Delft University of Technology

\title{
Spin Hall magnetoresistance in a canted ferrimagnet
}

Ganzhorn, Kathrin; Barker, Joseph; Schlitz, Richard; Piot, Benjamin A.; Ollefs, Katharina; Guillou, Francois; Wilhelm, Fabrice; Rogalev, Andrei; Bauer, Gerrit E W; More Authors

DOI

10.1103/PhysRevB.94.094401

Publication date

2016

Document Version

Final published version

Published in

Physical Review B (Condensed Matter and Materials Physics)

\section{Citation (APA)}

Ganzhorn, K., Barker, J., Schlitz, R., Piot, B. A., Ollefs, K., Guillou, F., Wilhelm, F., Rogalev, A., Bauer, G. E. W., \& More Authors (2016). Spin Hall magnetoresistance in a canted ferrimagnet. Physical Review B (Condensed Matter and Materials Physics), 94(9), 1-6. [094401].

https://doi.org/10.1103/PhysRevB.94.094401

Important note

To cite this publication, please use the final published version (if applicable).

Please check the document version above.

\section{Copyright}

Other than for strictly personal use, it is not permitted to download, forward or distribute the text or part of it, without the consent of the author(s) and/or copyright holder(s), unless the work is under an open content license such as Creative Commons.

\section{Takedown policy}

Please contact us and provide details if you believe this document breaches copyrights.

We will remove access to the work immediately and investigate your claim. 


\title{
Spin Hall magnetoresistance in a canted ferrimagnet
}

\author{
Kathrin Ganzhorn, ${ }^{1,2}$ Joseph Barker, ${ }^{3}$ Richard Schlitz, ${ }^{1,2}$ Benjamin A. Piot, ${ }^{4}$ Katharina Ollefs, ${ }^{5,6}$ Francois Guillou, ${ }^{5}$ \\ Fabrice Wilhelm, ${ }^{5}$ Andrei Rogalev, ${ }^{5}$ Matthias Opel, ${ }^{1}$ Matthias Althammer, ${ }^{1,2}$ Stephan Geprägs, ${ }^{1}$ Hans Huebl, ${ }^{1,2,7}$ \\ Rudolf Gross, ${ }^{1,2,7}$ Gerrit E. W. Bauer, ${ }^{3,8,9}$ and Sebastian T. B. Goennenwein ${ }^{1,2,7}$,* \\ ${ }^{1}$ Walther-Meißner-Institut, Bayerische Akademie der Wissenschaften, 85748 Garching, Germany \\ ${ }^{2}$ Physik-Department, Technische Universität München, 85748 Garching, Germany \\ ${ }^{3}$ Institute for Materials Research, Tohoku University, Sendai, Miyagi 980-8577, Japan \\ ${ }^{4}$ Laboratoire National des Champs Magnétiques Intenses, LNCMI-CNRS-UGA-UPS-INSA-EMFL, F-38042 Grenoble, France \\ ${ }^{5}$ European Synchrotron Radiation Facility (ESRF), 38043 Grenoble Cedex 9, France \\ ${ }^{6}$ Faculty of Physics and Center for Nanointegration Duisburg-Essen (CENIDE), Universität Duisburg-Essen, D-47057 Duisburg, Germany \\ ${ }^{7}$ Nanosystems Initiative Munich (NIM), 80799 München, Germany \\ ${ }^{8}$ Kavli Institute of NanoScience, Delft University of Technology, 2628 CJ Delft, The Netherlands \\ ${ }^{9}$ WPI Advanced Institute for Materials Research, Tohoku University, Sendai 980-8577, Japan
}

(Received 24 May 2016; published 1 September 2016)

\begin{abstract}
We study the spin Hall magnetoresistance effect in ferrimagnet/normal metal bilayers, comparing the response in collinear and canted magnetic phases. In the collinear magnetic phase, in which the sublattice magnetic moments are all aligned along the same axis, we observe the conventional spin Hall magnetoresistance. In contrast, in the canted phase, the magnetoresistance changes sign. Using atomistic spin simulations and x-ray absorption experiments, we can understand these observations in terms of the magnetic field and temperature dependent orientation of magnetic moments on different magnetic sublattices. This enables a magnetotransport based investigation of noncollinear magnetic textures.
\end{abstract}

DOI: 10.1103/PhysRevB.94.094401

The magnetic properties of ferromagnets are often modeled in terms of a simple macrospin with magnetization vector M. In this picture, one tacitly assumes that all individual atomic magnetic moments $\boldsymbol{\mu}$ are aligned in one direction, such that the magnetization is $\mathbf{M}=n \boldsymbol{\mu}$ with the moment number density $n$. However, many magnets exhibit a much richer magnetic structure, with canted, spiral, frustrated, or even topological [1,2] phases appearing in addition to collinear magnetic order. Unravelling these experimentally typically requires sophisticated methods, e.g., spin polarized neutron scattering, x-ray magnetic circular dichroism, or Lorentz transmission electron microscopy. A pathway for the electrical detection of magnetic properties is provided by spin torques arising at a magnet/metal interface [3-5]. These torques govern fundamental spintronic phenomena such as spin pumping [6-10], spin Seebeck effect [11-13], as well as spin Hall magnetoresistance [14-18], and even enable an electrical control of the magnetization in magnetic nanostructures [3-5]. However, while the spin torque effect—or more precisely the transfer of spin angular momentum across the magnet/metal interface-has been extensively discussed for a macrospin M [19,20], the action of spin torques on noncollinear magnetic phases is only poorly understood.

Here we show that in the ferrimagnet gadolinium iron garnet $\left(\mathrm{Gd}_{3} \mathrm{Fe}_{5} \mathrm{O}_{12}, \mathrm{GdIG}\right)$, the spin Hall magnetoresistance (SMR) can be used to resolve the orientation of magnetic moments residing on different magnetic sublattices. We thereby prove that the SMR is not just governed by the net moment $\boldsymbol{\mu}_{\text {net }}=\sum \boldsymbol{\mu}$ (viz. the corresponding macrospin magnetization

\footnotetext{
*Present address: Institut für Festkörperphysik, Technische Universität Dresden, D-01062 Dresden, Germany; goennenwein@ wmi.badw.de
}

$\mathbf{M}_{\text {net }}$ ) aligned along the externally applied magnetic field. This is reflected most conspicuously by the SMR sign inversion observed for canted sublattice moments. The interpretation of our experiments is corroborated by $\mathrm{x}$-ray magnetic circular dichroism (XMCD) measurements, and atomistic spin simulations [13] suggesting that the Fe sublattice moments dominate the SMR response.

The SMR originates from spin current transport across the interface between an (insulating) magnet and a metal with finite spin Hall angle. As sketched in Fig. 1(a), a charge current with density $\mathbf{J}_{\mathrm{c}} \| \mathbf{x}$ induces a spin current density with direction $\mathbf{J}_{\mathrm{s}}|| \mathbf{z}$ and polarization $\mathbf{s} \| \mathbf{y}$ in the metal. Depending on whether $\mathbf{J}_{\mathrm{s}}$ is absorbed or reflected at the interface, the metal's resistivity $\rho$ is either increased [Fig. 1(a)] or not [Fig. 1(b)]. In a collinear magnet, the amount of spin current at the interface can be modeled in terms of the magnetization direction $\mathbf{m}=\mathbf{M} / M=$ $\boldsymbol{\mu} / \mu$ relative to $\mathbf{s}$. As sketched in Fig. $1(\mathrm{c}), \boldsymbol{\mu} \perp \mathbf{s}$ corresponds to maximal spin transfer and thus large $\rho$, while $\boldsymbol{\mu} \| \mathbf{s}$ yields minimal $\rho$ [Fig. 1(d)], which can be parametrized by [14-16,21]

$$
\rho=\rho_{0}+\rho_{1}(\mathbf{m} \cdot \mathbf{y})^{2} .
$$

Here $\rho_{0}$ is the resistivity for $\mathbf{m} \| \mathbf{x}$. For more complex magnets, the use of Eq. (1) with $\mathbf{m}=\boldsymbol{\mu}_{\text {net }} / \mu_{\text {net }}$ becomes questionable. The magnet/metal exchange coupling in the SMR theory is formulated in terms of the spin mixing conductance, which for magnetic insulators is dominated by the local moments directly at the interface [22]. We can then illustrate the effect of the magnetization texture on the electron transport for a noncollinear magnet, viz. the ferrimagnetic insulator GdIG with three magnetic sublattices (FeA, FeD, and Gd) in a canted configuration as sketched in Fig. 1(e). Here none of the local moments $\boldsymbol{\mu}_{\mathrm{FeA}}, \boldsymbol{\mu}_{\mathrm{FeD}}$, and $\boldsymbol{\mu}_{\mathrm{Gd}}$ are parallel to $\boldsymbol{\mu}_{\mathrm{net}}$. Since the antiferromagnetic exchange coupling between the FeA and 
(a)

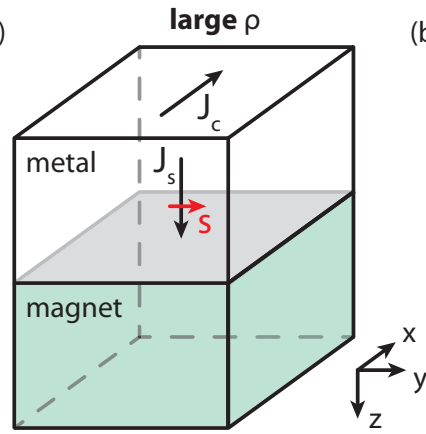

(c) collinear magnet large $\rho$

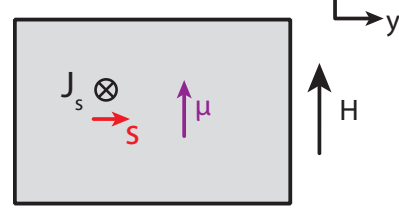

(e) canted magnet large $\rho$

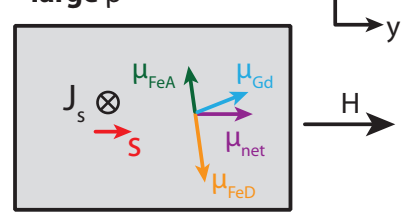

(f) canted magnet small $\rho$

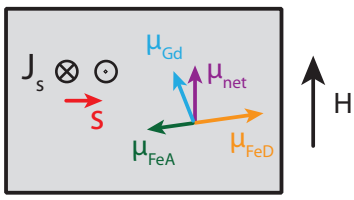

FIG. 1. Spin Hall magnetoresistance (SMR) response of a magnetic insulator/metal bilayer. (a) When the spin current $\mathbf{J}_{\mathrm{s}}$ in the metal is absorbed by the magnet, the resistivity $\rho$ of the metal is large. (b) When $\mathbf{J}_{\mathrm{s}}$ is reflected at the interface, $\rho$ is small owing to the inverse spin Hall effect. (c) and (d) In a collinear magnet, the spin transfer across the interface and thus $\rho$ is largest for $\boldsymbol{\mu} \perp \mathbf{s}$ (c), while spin transfer and $\rho$ are minimal for $\boldsymbol{\mu} \| \mathbf{s}$ (d). (e) and (f) In a noncollinear magnet in which, e.g., the orientation of the $\boldsymbol{\mu}_{\mathrm{FeA}}$ moments dominate the spin transfer across the interface, large viz. small $\rho$ arises for the corresponding orientations of $\boldsymbol{\mu}_{\mathrm{FeA}}$ with respect to $\mathbf{s}$. An externally applied magnetic field $\mathbf{H}$ (larger than the weak anisotropy but smaller than the interspin exchange fields) determines the orientation of $\boldsymbol{\mu}_{\text {net }}$. Comparing (c)-(f), the $\mathbf{H}$ orientations for maximum viz. minimum $\rho$ in the canted viz. collinear magnet are interchanged-the SMR inverts sign.

FeD moments is strong, we sketch them as antiparallel in the figure. Therefore, $\boldsymbol{\mu}_{\text {net }} \| \mathbf{H}$ is the vector sum of the net iron moment $\boldsymbol{\mu}_{\mathrm{Fe}}=\boldsymbol{\mu}_{\mathrm{FeD}}+\boldsymbol{\mu}_{\mathrm{FeA}}$ and of $\boldsymbol{\mu}_{\mathrm{Gd}}$.

To model the SMR in canted magnets, we assert that the spin-mixing conductance and the SMR are determined by the orientation of the individual, local magnetic moments $\mu_{X}$ at the interface. The SMR then reads

$$
\rho=\rho_{0}+\sum_{X} \rho_{1, X}\left\langle\left(\mathbf{m}_{X} \cdot \mathbf{y}\right)^{2}\right\rangle,
$$

where $\langle\cdots\rangle$ denotes the average over all moments of type $X$, and $\rho_{1, X}$ is the corresponding SMR resistivity modulation. For magnets with a collinear magnetization configuration, in which all sublattice moments are aligned parallel or antiparallel to each other, Eq. (2) is equivalent to Eq. (1) with $\rho_{1}=\sum_{X} \rho_{1, X}$.

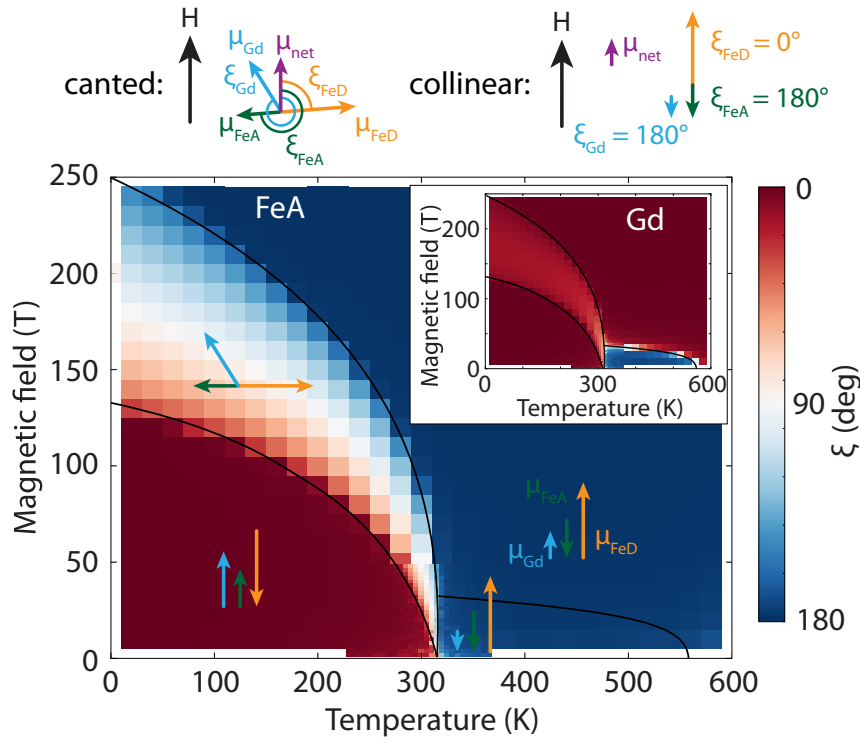

FIG. 2. Magnetic phase diagram of GdIG calculated by atomistic spin simulations (see text). The main panel depicts the orientation of the FeA sublattice moment orientation $\xi_{\mathrm{FeA}}$ encoded in color, the inset shows $\xi_{\mathrm{Gd}}$ of the Gd sublattice moments. Due to the strong antiferromagnetic exchange coupling, the FeD sublattice moments are always antiparallel to the $\mathrm{FeA}$ ones. The black lines indicate the temperature dependence of the upper $\left(\mu_{0} H_{c 2}\right)$ and lower $\left(\mu_{0} H_{c 1}\right)$ critical fields which delimit the antiparallel, parallel, and spin canting phase $[23,24]$. The orientation of the sublattice moments in each phase are represented by arrows.

In other words, the SMR response of a collinear ferrimagnet according to Eq. (2) looks exactly like the SMR of a simple, one-sublattice ferromagnet. In contrast, for magnets with noncollinear spin structure, the SMR response depends on the orientations of the different sublattice moments in a nontrivial way.

Most SMR experiments to date have been performed on bilayers made from yttrium iron garnet $\left(\mathrm{Y}_{3} \mathrm{Fe}_{5} \mathrm{O}_{12}, \mathrm{YIG}\right)$ as the insulating magnet and platinum $(\mathrm{Pt})$ as the metal. The magnetic properties of YIG stem from two octahedrally coordinated $\mathrm{Fe}^{3+}$ moments ( $\mathrm{FeA}$ ) and three tetrahedrally coordinated $\mathrm{Fe}^{3+}$ moments (FeD) per formula unit. The FeA and FeD moments are strongly antiferromagnetically coupled. YIG therefore is a collinear ferrimagnet, warranting the use of Eq. (1). Only in magnetic fields in excess of $\mu_{0} H_{c 1} \approx 250 \mathrm{~T}$, a canted magnetic phase emerges, in which $\mathbf{H}, \boldsymbol{\mu}_{\mathrm{FeA}}$, and $\boldsymbol{\mu}_{\mathrm{FeD}}$ are no longer aligned along one common axis [1,23-25].

In contrast to YIG, the canted magnetic phase is readily accessible in compensated magnetic garnets such as GdIG, see Fig. 2. Due to their exchange coupling to the FeA and $\mathrm{FeD}$ moments, the paramagnetic Gd moments acquire a finite sublattice magnetization [1]. We model the GdIG magnetic structure using a classical Heisenberg Hamiltonian including all of the atoms in the unit cell (see Ref. [13] for details of the model). We use a Metropolis Monte Carlo algorithm with a combination of different moves to avoid trapping in metastable minima [26], to calculate the equilibrium magnetic configuration as a function of applied field and temperature, disregarding the small crystalline anisotropy. 
The system size is $16 \times 16 \times 16$ unit cells (131 072 spins) with periodic boundary conditions. In particular, we take the spin configuration at the surface to be similar to that of the bulk. Figure 2 shows the (average) orientation $\xi_{\mathrm{FeA}}$ of the FeA sublattice moments with respect to the applied field direction in the main panel, as well as the orientation $\xi_{\mathrm{Gd}}$ of the $\mathrm{Gd}$ ones in the inset. Since the FeA and $\mathrm{FeD}$ moments are coupled via a strong antiferromagnetic exchange, $\xi_{\mathrm{FeD}}=\xi_{\mathrm{FeA}}+180^{\circ}$. We note that since different magnetic textures can result in the same $\boldsymbol{\mu}_{\mathrm{net}}$, atomistic modeling is necessary to establish the SMR as a function of magnetic field and temperature. As evident from Fig. 2, the FeA, FeD, and Gd sublattices arrange in different configurations depending on temperature and external magnetic field. Moreover, a magnetic compensation point with $\mathbf{M}_{\text {net }}=0$ for $H=0$ arises at the so-called compensation temperature $T_{\text {comp }} \approx 300 \mathrm{~K}$. A canted magnetic phase is easily accessible already for magnetic fields of a few Tesla in the vicinity of $T_{\text {comp. }} T_{\text {comp }}$ and the critical fields are reduced by alloying In and Y into GdIG $[1,27]$, so that a large portion of the canted phase becomes accessible using standard magnet cryostats. SMR experiments in InYGdIG/Pt bilayers thus are an ideal testbed to check the validity of Eq. (2).

We here discuss experiments on two different garnet/Pt bilayers. The magnetic garnet layers were deposited onto single crystalline, [111]-oriented yttrium aluminum garnet $\left(\mathrm{Y}_{3} \mathrm{Al}_{5} \mathrm{O}_{12}, \mathrm{YAG}\right)$ substrates via pulsed laser deposition (PLD). The yttrium iron garnet $\left(\mathrm{Y}_{3} \mathrm{Fe}_{5} \mathrm{O}_{12}\right.$, YIG) film was grown using a substrate temperature of $500^{\circ} \mathrm{C}$, an oxygen atmosphere of $2.5 \times 10^{-2} \mathrm{mbar}$, and an energy fluence of the $\mathrm{KrF}$ excimer laser of $2.0 \mathrm{~J} / \mathrm{cm}^{2}$ at the target surface. The 40-nm-thick YIG film was covered in situ with $t=4 \mathrm{~nm}$ of Pt deposited via electron beam evaporation. The same growth parameters were used for the indium and yttrium doped gadolinium iron garnet $\left(\mathrm{Y}_{1} \mathrm{Gd}_{2} \mathrm{Fe}_{4} \mathrm{In}_{1} \mathrm{O}_{12}\right.$, InYGdIG) film, which has a thickness of $61.5 \mathrm{~nm}$ and was covered with $t=3.6 \mathrm{~nm}$ of Pt. In previous experiments, we found that YIG films with thicknesses in the range of 20 to $100 \mathrm{~nm}$ have very similar magnetic properties. We therefore consider the somewhat different physical thickness of the YIG and InYGdIG films unimportant in the present study. The InYGdIG sample exhibits a magnetization compensation temperature $T_{\text {comp }}=85 \mathrm{~K}$, such that magnetotransport experiments at temperatures well above and well below $T_{\text {comp }}$ are possible in our magnet cryostat system.

The magnetic properties of the InYGdIG sample, in particular the orientation of the individual magnetic sublattices close to $T_{\text {comp }}$, were investigated via element-specific XMCD measurements performed at the European Synchrotron Radiation Facility (ESRF) at the beamline ID12 using the total fluorescence yield (TFY) detection mode. The sample was mounted on a cold finger cryostat $(2.1 \leqslant T \leqslant 300 \mathrm{~K})$ and inserted in the bore of a superconducting magnet allowing for magnetic fields up to $17 \mathrm{~T}$. X-ray absorption near edge spectra (XANES) were recorded at the $\mathrm{Fe} K$ edge [28] with right and left circularly polarized light, as well as positive and negative in plane magnetic fields. The XANES taken at $17 \mathrm{~T}$ and $50 \mathrm{~K}$ is shown in Fig. 3(a). The pre-edge at $7114 \mathrm{eV}$ marked by a black arrow arises mainly from the tetrahedrally ordered FeD sublattice [29]. Since the FeA moments are antiparallel
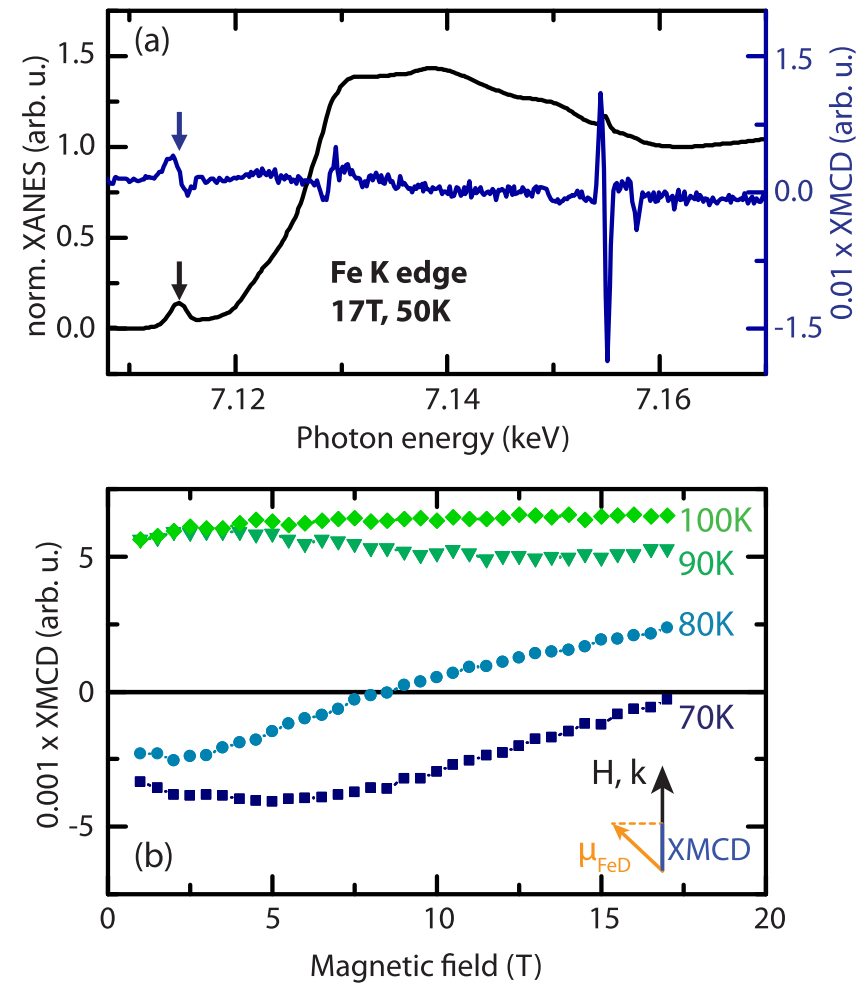

FIG. 3. (a) Normalized XANES recorded at the Fe $K$ edge in the InYGdIG/Pt sample at $50 \mathrm{~K}$ and $17 \mathrm{~T}$. The pre-edge marked by a black arrow arises mainly from the tetrahedrally ordered FeD moments. The blue curve represents the corresponding XMCD signal, which is dominated by the signal at the pre-edge. (b) XMCD amplitude, i.e., the projection of the FeD moments onto the external field axis, measured as a function of field strength at various temperatures around $T_{\text {comp }}$.

to the FeD moments for the magnetic field accessible in our experiments as also shown in the atomistic simulations, it is sufficient to analyze the pre-edge. Several XANES were recorded to improve the signal-to-noise ratio and normalized to an edge jump of unity. The XMCD signal was calculated as the direct difference between consecutive normalized XANES recorded either with right and left circularly polarized light or while applying positive and negative magnetic fields. The resulting XMCD spectrum at $17 \mathrm{~T}$ and $50 \mathrm{~K}$ is also depicted in Fig. 3(a). Note that the XMCD measurements give access to the projection of the magnetization onto the $k$ vector of the incoming x-ray beam (viz. external magnetic field direction). Therefore, a rotation of the FeD sublattice orientation with respect to the external field from a parallel to an antiparallel configuration, as expected in a compensated ferrimagnet close to $T_{\text {comp }}$ (see Fig. 2), will manifest itself as a sign change of the pre-edge XMCD signal.

In order to map out the canted phase in InYGdIG, the $\mathrm{XMCD}$ signal at the $\mathrm{Fe} K$ pre-edge was measured as a function of magnetic field for temperatures between 70 and $100 \mathrm{~K}$. For a quantitative evaluation of the XMCD signal, the peak to peak amplitude of the pre-edge XMCD signal was calculated as the difference between the extrema at 7115 and $7114 \mathrm{eV} \mathrm{[30].}$ As shown in Fig. 3(b), the XMCD amplitude is positive and independent of field strength for $T=100 \mathrm{~K}$ and $90 \mathrm{~K}>T_{\text {comp }}$, indicating that the $\mathrm{FeD}$ moments are saturated and parallel 
to the external field. For $T=80 \mathrm{~K}$, however, the XMCD amplitude changes sign as a function of magnetic field strength, with positive amplitude at high magnetic fields and negative amplitude at low magnetic fields. Finally, for $T=70 \mathrm{~K}$, the $\mathrm{XMCD}$ amplitude is negative for all fields studied. This sign change of the XMCD amplitude as a function of temperature and magnetic field strength provides clear evidence for the reorientation of the $\mathrm{FeD}$ sublattice around the compensation temperature, which was determined to $T_{\text {comp }}=85 \mathrm{~K}$ using SQUID magnetometry measurements in this sample. Note also that the zero crossings of the XMCD amplitude correspond to a perpendicular orientation of the FeD sublattice with respect to the external field, i.e., to the white region in Fig. 2. The XMCD measurements in particular confirm that the InYGdIG sample studied here features all the characteristic magnetic properties of a compensated ferrimagnet, i.e., a compensation temperature and a canting phase.

For the magnetoresistance experiments, the InYGdIG/Pt and YIG/Pt bilayers were patterned into Hall bars with width $w=80 \mu \mathrm{m}$ and length $l=600 \mu \mathrm{m}$ using optical lithography and argon ion beam milling. The samples were mounted in the variable temperature insert of a superconducting magnet cryostat $(10 \leqslant T \leqslant 300 \mathrm{~K})$ at the Walther-Meissner-Institut (WMI) for measurements at magnetic fields up to $\mu_{0} H=7 \mathrm{~T}$. Additional measurements up to $\mu_{0} H=29 \mathrm{~T}$ were conducted using a resistive magnet setup with a variable temperature insert at the high-field magnet laboratory in Grenoble. In both setups, a constant current of $I=0.2 \mathrm{~mA}$ was applied along the Hall bar using a Keithley 2400 sourcemeter. We carried out angle-dependent magnetoresistance measurements [15] by rotating the sample with respect to the external magnetic field of fixed magnitude $\mu_{0} H \leqslant 29 \mathrm{~T}$ applied in the sample plane, simultaneously recording the voltage drop $V$ along the direction of charge current as a function of the angle $\alpha_{H}$ between the current direction $\mathbf{J}_{\mathrm{c}}$ and the external magnetic field $\mathbf{H}$ using a Keithley 2182 nanovoltmeter. Hereby we used a current reversal method in order to cancel thermopower effects and reduce noise.

Figures 4(a)-4(c) show a typical set of magnetoresistance measurements for the YIG/Pt bilayer, taken at fixed temperatures of $T=10,85$, and $300 \mathrm{~K}$ and an external magnetic field of $7 \mathrm{~T}$. The magnetoresistance behavior is fully consistent with previous measurements [15,31]. Indeed, taking both the applied magnetic field $\mathbf{H}$ and $\mathbf{m}$ to reside in the magnet/metal interface plane (the $\mathbf{x y}$ plane in Fig. 1), Eq. (1) can be rewritten as $\rho\left(\alpha_{H}\right)=\rho_{0}+\rho_{1} \sin ^{2} \alpha_{H}$, with $\rho_{1}<0$ [21]. The SMR amplitude

$$
\frac{-\rho_{1}}{\rho\left(\alpha_{H}=0^{\circ}\right)}=\frac{\rho\left(\alpha_{H}=0^{\circ}\right)-\rho\left(\alpha_{H}=90^{\circ}\right)}{\rho\left(\alpha_{H}=0^{\circ}\right)}
$$

is positive at all temperatures, and decreases with decreasing temperature by about a factor of 2 as also reported in the literature [31]. A similar set of magnetoresistance measurements for the InYGdIG/Pt sample is depicted in Figs. 4(d)-4(f), again for $T=10,85$, and $300 \mathrm{~K}$. The measurements at $T=10 \mathrm{~K}$ and $T=300 \mathrm{~K}$ [Figs. 4(d) and 4(f)] show the same positive SMR as for YIG/Pt. However, at $T=85 \mathrm{~K} \approx T_{\text {comp }}$ [Fig. 4(e)], the SMR has negative sign, and comparatively small amplitude. This is surprising and cannot be accounted for by the standard SMR theory as written in Eq. (1) $[16,21]$.
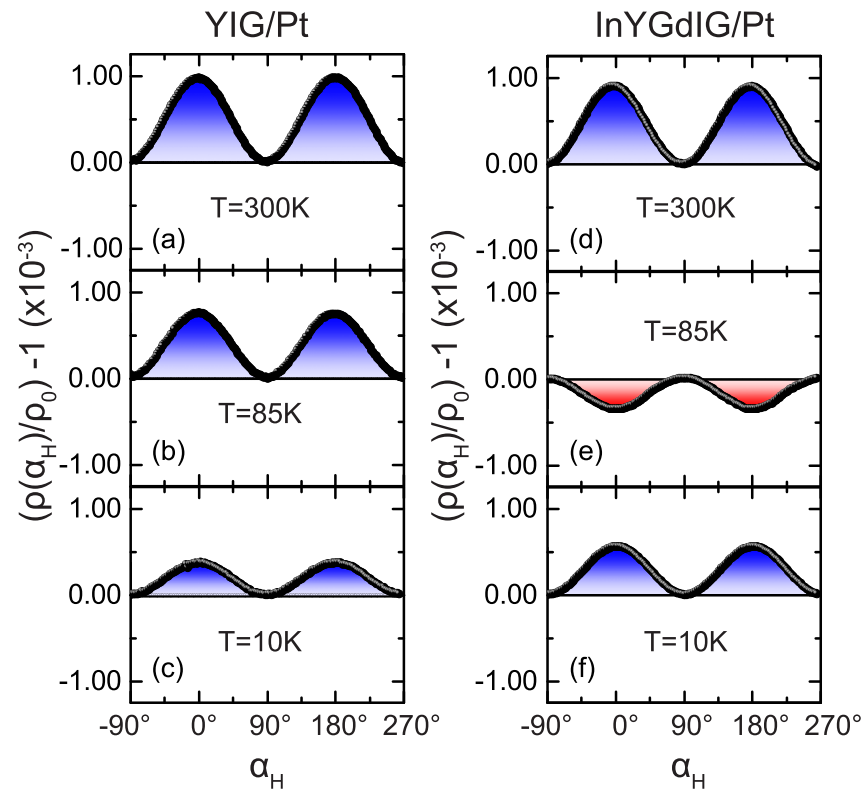

FIG. 4. Measured evolution of the magnetoresistance in YIG/Pt (a)-(c) and InYGdIG/Pt (d)-(f). The data were recorded at $T=10$, 85 , and $300 \mathrm{~K}$ as a function of the angle $\alpha_{H}$ between the current direction $\mathbf{J}_{c}$ and the orientation of the external, in-plane magnetic field $\mu_{0} H=7 \mathrm{~T}$. The SMR in InYGdIG/Pt inverts sign around the magnetization compensation temperature $T_{\text {comp }} \approx 85 \mathrm{~K}$ (e), but the extrema stay at the same $\alpha_{H}$ for all temperatures.

To substantiate the SMR sign change, we studied the evolution of the SMR amplitude [Eq. (3)] with magnetic field strength and temperature in the YIG/Pt and InYGdIG/Pt samples. In YIG/Pt, the SMR amplitude monotonically increases with $T$, as reported previously [31]. In InYGdIG/Pt, the behavior is much richer. Figure 5 shows corresponding data obtained for $\mu_{0} H \leqslant 7 \mathrm{~T}$ in the superconducting magnet cryostat at WMI, as well as $\mu_{0} H \leqslant 29 \mathrm{~T}$ at the high field magnet laboratory in Grenoble, in a false color plot. The SMR

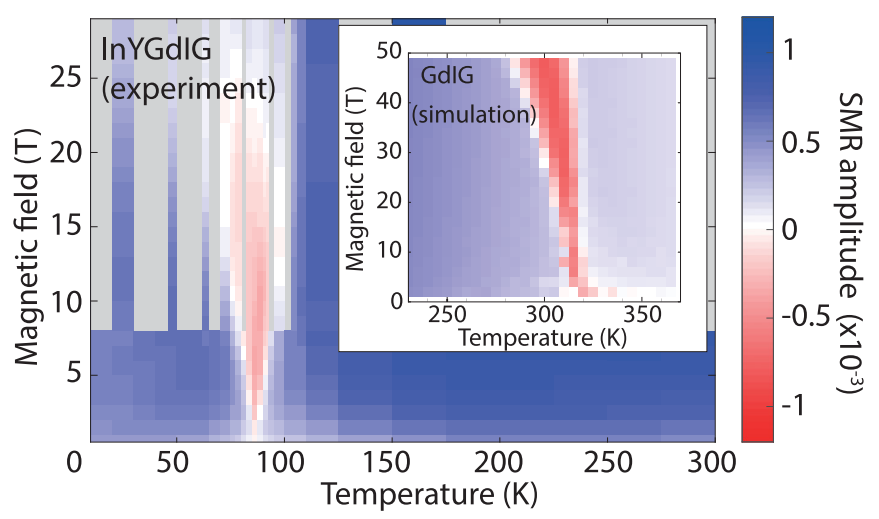

FIG. 5. SMR amplitude Eq. (3) as a function of temperature and magnetic field, as measured for InYGdIG (main figure), and calculated for GdIG (inset) only taking the iron moments into account. In the blue regions the SMR is positive, i.e., has the same sign and $\alpha_{H}$ dependence as for a single-sublattice ferromagnet [cf. Fig. 1(a)]. The red regions indicate negative SMR [as in Fig. 4(e)]. No data has been taken in the regions shaded in gray. 
sign change in InYGdIG/Pt is clearly evident as a red pocket around $T_{\text {comp }}($ InYGdIG $)=85 \mathrm{~K}$.

We may conclude with confidence that the macrospin picture of the SMR breaks down for noncollinear magnets. Since the spin current transport across the magnetic insulator/normal metal interface relevant for SMR corresponds to an additional (transverse) dissipation channel for charge transport, $\rho_{1}<0$ cannot change sign with temperature $[15,16]$. The large external magnetic fields well exceed the demagnetizing or anisotropy fields, such that the orientation $\alpha_{H}$ of $\mu_{0} H$ is identical to the orientation of $\boldsymbol{\mu}_{\text {net }}$ viz. $\mathbf{M}_{\text {net }}$. Thus, if $\mathbf{m}=\boldsymbol{\mu}_{\text {net }} / \mu_{\text {net }}$ indeed would govern the SMR in the spirit of Eq. (1), the SMR amplitude should be positive for all temperatures and magnetic fields. The InYGdIG/Pt sample clearly violates this conjecture, showing that the SMR is a powerful method to characterize complex spin textures. The small SMR modulation observed in $\mathrm{CoCr}_{2} \mathrm{O}_{4}$ and $\mathrm{Cu}_{2} \mathrm{OSeO}_{3}$ can thus indeed be evidence for different spin spiral phases $[32,33]$.

In the following we show that the SMR response summarized in Fig. 5 can be straightforwardly understood assuming that the magnetic sublattice moments contribute independently to the SMR, as expressed in Eq. (2). Since the exchange parameters of InYGdIG are not well known, we compare the experimental SMR data from InYGdIG/Pt with the SMR calculated for GdIG/Pt (Fig. 5). While the compensation temperatures of GdIG and InYGdIG are different, the spin correlations and thus the canted phases should be qualitatively similar. Indeed, the SMR response calculated from Eqs. (2) and (3) using the sublattice moment orientations $\xi_{X}$ from Fig. 2 reproduces all the salient features observed in experiment. Interestingly, a reasonable agreement between model and experiment is obtained already upon taking into account only the iron moments, as shown in the inset of Fig. 5. The Gd moments play a minor role for the SMR in GdIG, owing to a large spread in their directions arising from thermal fluctuations. Assuming that (only) the iron sublattice moment orientations govern the SMR, we can understand its sign reversal in the canted phase from Fig. 1. While in the collinear phase the iron (and also the Gd) moments are aligned along the $\mathbf{H}$ axis, they rotate away from $\mathbf{H}$ in the canted phase. As indicated in the figure, this reorientation of the iron magnetic moments relative to the applied magnetic field causes the inversion of the SMR. The magnetic field and temperature range in which this inversion takes place, is consistent with the reorientation of the FeD moments observed in the XMCD experiments (see Fig. 3). A conventional positive SMR amplitude is expected when the iron moments are aligned or anti-aligned with the magnetic field direction, i.e., in the regions shaded red and blue in the main panel of Fig. 2. The SMR amplitude is inverted when the iron moments are orthogonal to $\mathbf{H}$, corresponding to the white regions in Fig. 2. This simple picture matches the experimental observations well.

Note that in the collinear state at high temperatures, our SMR measurements up to $29 \mathrm{~T}$ show no indication of a field suppression in contrast to the one observed in SSE experiments in YIG [34]. This is consistent with the SMR description based on spin transfer torque [16].

We also would like to emphasize that the SMR sign changes discussed here are qualitatively different from those observed in SSE experiments in GdIG/Pt heterostructures [13]. While the sign change of the SMR is found only in the canting phase, Geprägs $e t$ al. report a second sign change of the SSE at low temperatures due to the contribution of multiple magnon branches [13]. These observations are consistent with our physical picture that the SMR reflects the orientation of the $\mathrm{Fe}$ sublattice moments and is therefore independent of magnon modes.

In summary, we observe a sign inversion of the SMR in compensated ferrimagnet/Pt bilayers around $T_{\text {comp }}$. We attribute this behavior to the noncollinear reorientation of the sublattice moments in the spin canting phase, as corroborated by complementary XMCD measurements. We show that the experimental data can be understood assuming that the magnetic moments in the different magnetic sublattices contribute independently to the SMR. Our results demonstrate that simple transport experiments can identify noncollinear magnetic phases in highly resistive magnets contacted by heavy metals. The SMR thus might prove useful also for the investigation of topological spin textures, e.g., skyrmions, in thin films and nanostructures.

We thank Francesco Della Coletta, Sibylle Meyer, and Sascha Frölich for sample fabrication and gratefully acknowledge financial support via DFG Priority Programme 1538 Spin-Caloric Transport (GO 944/4, BA 2954/2), ESRF Project HC-2058, EU FP7 ICT Grant No. 612759 InSpin, and Grant-in-Aid for Scientific Research (Grants No. 25247056, No. 25220910, No. 26103006). J.B. acknowledges support from the Graduate Program in Spintronics, Tohoku University.
[1] G. F. Dionne, Magnetic Oxides (Springer, New York, 2009).

[2] S. Mühlbauer, B. Binz, F. Jonietz, C. Pfleiderer, A. Rosch, A. Neubauer, R. Georgii, and P. Böni, Science 323, 915 (2009).

[3] S. I. Kiselev, J. C. Sankey, I. N. Krivorotov, N. C. Emley, R. J. Schoelkopf, R. A. Buhrman, and D. C. Ralph, Nature (London) 425, 380 (2003).

[4] I. M. Miron, G. Gaudin, S. Auffret, B. Rodmacq, A. Schuhl, S. Pizzini, J. Vogel, and P. Gambardella, Nat. Mater. 9, 230 (2010).

[5] S. Emori, U. Bauer, S.-M. Ahn, E. Martinez, and G. S. D. Beach, Nat. Mater. 12, 611 (2013).

[6] R. Urban, G. Woltersdorf, and B. Heinrich, Phys. Rev. Lett. 87, 217204 (2001).
[7] Y. Tserkovnyak, A. Brataas, and G. E. W. Bauer, Phys. Rev. Lett. 88, 117601 (2002).

[8] O. Mosendz, J. E. Pearson, F. Y. Fradin, G. E. W. Bauer, S. D. Bader, and A. Hoffmann, Phys. Rev. Lett. 104, 046601 (2010).

[9] F. D. Czeschka, L. Dreher, M. S. Brandt, M. Weiler, M. Althammer, I.-M. Imort, G. Reiss, A. Thomas, W. Schoch, W. Limmer, H. Huebl, R. Gross, and S. T. B. Goennenwein, Phys. Rev. Lett. 107, 046601 (2011).

[10] S. Takei, T. Moriyama, T. Ono, and Y. Tserkovnyak, Phys. Rev. B 92, 020409 (2015).

[11] K. Uchida, H. Adachi, T. Ota, H. Nakayama, S. Maekawa, and E. Saitoh, Appl. Phys. Lett. 97, 172505 (2010). 
[12] M. Weiler, M. Althammer, F. D. Czeschka, H. Huebl, M. S. Wagner, M. Opel, I.-M. Imort, G. Reiss, A. Thomas, R. Gross, and S. T. B. Goennenwein, Phys. Rev. Lett. 108, 106602 (2012).

[13] S. Geprägs, A. Kehlberger, F. D. Coletta, Z. Qiu, E.-J. Guo, T. Schulz, C. Mix, S. Meyer, A. Kamra, M. Althammer, H. Huebl, G. Jakob, Y. Ohnuma, H. Adachi, J. Barker, S. Maekawa, G. E. W. Bauer, E. Saitoh, R. Gross, S. T. B. Goennenwein, and M. Klaui, Nat. Commun. 7, 10452 (2016).

[14] H. Nakayama, M. Althammer, Y.-T. Chen, K. Uchida, Y. Kajiwara, D. Kikuchi, T. Ohtani, S. Geprägs, M. Opel, S. Takahashi, R. Gross, G. E. W. Bauer, S. T. B. Goennenwein, and E. Saitoh, Phys. Rev. Lett. 110, 206601 (2013).

[15] M. Althammer, S. Meyer, H. Nakayama, M. Schreier, S. Altmannshofer, M. Weiler, H. Huebl, S. Geprägs, M. Opel, R. Gross, D. Meier, C. Klewe, T. Kuschel, J.-M. Schmalhorst, G. Reiss, L. Shen, A. Gupta, Y.-T. Chen, G. E. W. Bauer, E. Saitoh, and S. T. B. Goennenwein, Phys. Rev. B 87, 224401 (2013).

[16] Y.-T. Chen, S. Takahashi, H. Nakayama, M. Althammer, S. T. B. Goennenwein, E. Saitoh, and G. E. W. Bauer, Phys. Rev. B 87, 144411 (2013).

[17] C. Hahn, G. de Loubens, O. Klein, M. Viret, V. V. Naletov, and J. Ben Youssef, Phys. Rev. B 87, 174417 (2013).

[18] N. Vlietstra, J. Shan, V. Castel, B. J. van Wees, and J. Ben Youssef, Phys. Rev. B 87, 184421 (2013).

[19] J. C. Slonczewski, J. Magn. Magn. Mater. 159, L1 (1996).

[20] L. Berger, Phys. Rev. B 54, 9353 (1996).
[21] Y.-T. Chen, S. Takahashi, H. Nakayama, M. Althammer, S. T. B. Goennenwein, E. Saitoh, and G. E. W. Bauer, J. Phys.: Condens. Matter 28, 103004 (2016).

[22] X. Jia, K. Liu, K. Xia, and G. E. W. Bauer, Europhys. Lett. 96, 17005 (2011).

[23] J. Bernasconi and D. Kuse, Phys. Rev. B 3, 811 (1971).

[24] A. E. Clark and E. Callen, J. Appl. Phys. 39, 5972 (1968).

[25] N. Miura, I. Oguro, and S. Chikazumi, J. Phys. Soc. Jpn. 45, 1534 (1978).

[26] D. Hinzke and U. Nowak, Comput. Phys. Commun. 121-122, 334 (1999).

[27] J. R. Cunningham and E. E. Anderson, J. Appl. Phys. 32, S388 (1961).

[28] A. Rogalev, J. Goulon, F. Wilhelm, C. Brouder, A. Yaresko, J. B. Youssef, and M. Indenbom, J. Magn. Magn. Mater. 321, 3945 (2009).

[29] H. Maruyama and N. Kawamura, J. Electron Spectrosc. Relat. Phenom. 136, 135 (2004).

[30] C. Strohm, T. Roth, C. Detlefs, P. van der Linden, and O. Mathon, Phys. Rev. B 86, 214421 (2012).

[31] S. Meyer, M. Althammer, S. Geprägs, M. Opel, R. Gross, and S. T. B. Goennenwein, Appl. Phys. Lett. 104, 242411 (2014).

[32] A. Aqeel, N. Vlietstra, J. A. Heuver, G. E. W. Bauer, B. Noheda, B. J. van Wees, and T. T. M. Palstra, Phys. Rev. B 92, 224410 (2015).

[33] A. Aqeel, N. Vlietstra, A. Roy, M. Mostovoy, B. J. van Wees, and T. T. M. Palstra, arXiv:1607.05630.

[34] T. Kikkawa, K.-i. Uchida, S. Daimon, Z. Qiu, Y. Shiomi, and E. Saitoh, Phys. Rev. B 92, 064413 (2015). 